\title{
Brief Analysis of Application of Objective Structured Clinical Examination (OSCE) in Graduation Exams of Clinical Medical Students
}

\author{
Yihua Du, Ke Yu, Xiaohong Li, Feng Wang \& Tingting Wang \\ Affiliated Hospital of Clinical Medical School, LuZhou Medical College \\ Luzhou 646000, Sichuan, China
}

Received: July 21, 2011

Accepted: June 26, $2011 \quad$ Published: December 1, 2011

doi:10.5539/hes.v1n2p92

URL: http://dx.doi.org/10.5539/ hes.v1n2p92

\begin{abstract}
This article gives a brief introduction to the Objective Structured Clinical Examination (OSCE) and analyzes developmental progress of OSCE at both home and abroad and standardized patients' application in OSCE. Also, this article expounds application of OSCE in graduation exam of clinical medical students. Finally, this article summarizes pre-exam preparation work and flow of OSCE exam in Affiliated Hospital of Clinical Medical School, analyzes OSCE and puts forward suggestions to improve OSCE.
\end{abstract}

Keywords: OSCE, Graduation exam, Clinical medical students

Clinical medicine is a subject with strong practicality and its cultivation target is to enable medical students to learn to become application-oriented medical talents with independent clinical working capacity. Establishment of an effective, scientific and rational clinical practical skill evaluation system is of extremely important significance to the learning purpose and method of students. Previous clinical medical teaching evaluation is deficient in objective clinical practical skill evaluation system, which causes students' learning focus usually to be placed upon "theoretical" knowledge in learning clinical skills, whereas practical clinical skill usually does not conform with the score of evaluation and is unable to adapt rapidly to the independent clinical work after graduation.

In 2010, Affiliated Hospital of Clinical Medical School organized a graduation exam involving nearly 700 students among medical undergraduates with the OSCE mode. Here, we are going to present application situation and major problems that need to be improved in OSCE.

\section{Summary of OSCE Exam}

OSCE (Objective Structured Clinical Examination) is a sort of evaluation method which was proposed by Dr. R.M. Harden from Dundee University in Britain in 1975, in which an objective method was used to evaluate clinical working capacity, and its basic idea is "a test based on operation". OSCE is a kind of evaluation method which uses a model, standardized patients and even patients to test the clinical working capacity of medical students in the situation of simulating a clinical occasion. Simultaneously, it is a sort of method of comprehensive capacity evaluation with equal emphasis of knowledge, skill and attitude. OSCE is constituted by multiple "stations", each station aimed at different exam points and evaluating more knowledge and capacity of students. OSCE reflects the evaluation model based on capacity and has important orientation effect upon education and cultivation of medical students.

\section{Developmental Progress of OSCE at both Home and Abroad}

In the 90s of the Twentieth Century, US applied OSCE into exams which were aimed at foreign medical graduates' qualification authentication. In 2002, US again applied OSCE into the second stage of Qualification Examination for Doctors. According to curriculum arrangement of US medical colleges, $96 \%$ colleges used the pattern of different standardized patients (SP) to evaluate clinical skills and 60\% colleges applied OSCE into evaluation upon clinical capacity of medical students. In the US Qualification Examination for Doctors, there are altogether 12 stations in the OSCE, each station with 25 minutes, in which standardized patients evaluation examinees' ability to collect and record patients' data and their communication skills as well as oral English capacity. Firstly, examinees read about patients' information and requirements of a single station, and then they are asked to have a fifteen-minute significant inquiry and physical quality examination, discussing together with patients about initial diagnosis and further diagnosis plan, responding to questions of patients and leaving the station (no longer returning) within fifteen minutes. Finally, examinees are required to record patients' disease history and positive as well as negative discoveries in the physical quality examination within ten minutes, propose diagnosis and differential diagnosis (at 
most five) and put forward further diagnosis plan. Canadian Medical Association applied OSCE into Medical Licensing Examination in 1993.

In 1990, China began to bring in OSCE teaching evaluation method. For example, Sichuan University (formerly West China University of Medical Sciences), Zhejiang University (formerly Zhejiang Medical University) and Jiujiang Medical School were sponsored by China Medical Board (CMB) in US, and Professor Paula L. Stillman guided in the teaching research of the clinical skill evaluation program, which acquired success.

\section{Application of Standardized Patients in OSCE}

In "International Standard for Undergraduate Medical Education of World Federation for Medical Education", it was pointed out that, medical college had to ensure that medical students could come into contact with patients, obtain enough clinical knowledge and skill and then assume appropriate clinical obligations after graduation. With continuous expansion of enrollment in medical colleges and schools and continuously enhanced consciousness of patients' in protection, quite a large number of patients are reluctant to allow internship doctors to have an examination on themselves, which will necessarily largely reduce the internship quality. Thus, it is urgently required to find out a sort of teaching mode to take the place of real patients so as to guarantee the internship quality of medical students and, thereby, the concept of standardized patients emerges at the right moment. Standardized patient is abbreviated as SP, which is to train volunteers recruited in a systematic way and simulate a certain kind of clinical case in the exam so as to evaluate the clinical skills of medical students. Standardized patients might be healthy people.

In Japan, Kawasaki medical university started to bring in OSCE in 1992. For the past several years, there have been almost 40 universities that have brought OSCE into their teaching plans. By the end of 1998, there had already been 15 SP training and activity centers in the whole country, which offered service for OSCE, clinical continuing education and medical education research. In 1991, Panla L Stillman introduced SP into China. In the SP exam station in China, standardized patients serve as the double roles of examiner and teacher. Since the standard for scoring is in great details, objectivity of scoring is guaranteed. Accuracy of SP in recording behaviors of testees is the key to whether OSCE is effective. China Medical University has conducted a study with the standard of scoring by professional clinicians. According to the result, the ability of SP to evaluate medical students is in consistency with the ability of professional clinicians to evaluation medical students, which ensures the accuracy of scoring.

Nevertheless, SP does not cover all fields in the clinical medicine, such as, physical examination in gynaecology, physical examination in paediatrics and positive symbols in quite a lot of physical examinations in medicine and surgery that can't be simulated, eg., ascites, spider angioma, jaundice and so on. Furthermore, it is generally reacted by medical students that there is a large gap between those that are simulated and those real patients in physical examination in gynaecology. Since gynaecology involves privacy of patients, currently, exam station in gynaecology can merely evaluation medical students' capacity in collecting disease history, but does not apply to operational check in gynaecology and obstetrics, which largely confines application of SP in gynaecology and obstetrics.

In Shanghai, Shanghai Medical College of Fudan University firstly started training on standardized patients. The first batch of 39 standardized patients obtained a certificate of competency and participated in skill evaluation on seven-year students in the clinical major.

\section{Pre-exam Preparation Work in OSCE in Luzhou Medical College}

\subsection{Leaders of the Hospital Attached Great Importance and Actively Planned Construction of OSCE Station and Training on Examiners}

4.1.1 From 2008 to 2009, LuZhou Medical College continuously explored an OSCE mode that was suitable for its own reality. Through visiting and inspecting domestic brother colleges and taking into consideration of the reality, the college formulated "OSCE Clinical Skill Evaluation System Establishment Plan in Clinical Medical Major of LuZhou Medical College" and "OSCE Clinical Skill Evaluation Plan in Clinical Medical Major of LuZhou Medical College (on trial)", established OSCE clinical skill evaluation leading groups and successively made an investment of more than 1 million RMB Yuan in construction of evaluation platform.

4.1.2 The college invited experts from outside (Sichuan University West China clinical skill experimental teaching center professors) to give lectures. These experts mainly taught about application of standardized patients in clinical skill teaching and evaluation, OSCE and so on. West China SP teachers also gave on-the-spot SP demonstration.

4.1.3 The college organized senior and professional backbone teachers to set up a question bank group, established a question bank according to teaching syllabus and basic requirements of clinical skills, carried out rational scoring index system, and controlled any variable factor to the best so as to improve the objectivity degree of scoring results and reduce influences of subjective factors on the scoring result. Furthermore, the college sent heads of the teaching 
and research section of the medicine, surgeon, gynaecology and paediatrics to walk out to learn successful experiences of OSCE in other colleges. The content of the question bank included cases of simulation, disease history-taking and case analysis, physical examination, diagnosis and treatment operational skill, reading of pictures and so on. Questions of different types were all given numbering so as to enable students to randomly select the test questions.

\subsection{Interaction between Teachers and Students and Pre-examination Propaganda}

The college notified students, in advance, of the examination flow and the do's and don'ts, analyzed the current examination situation of medical students and the situation of the Qualification Examination for Doctors they were to face up with in the future, explained necessity and importance of OSCE and encouraged students to prepare the exam carefully.

\subsection{The College Carried out Definite Working Plan and Working Flow}

In the multi-station examination, the number of students was large and the work of organization was complex. Before the examination began, first of all, a definite working plan and working flow had to be carried out, a basic idea was necessary about the arrangement of the number of students in each examination station, the time and the sequence of the examination, and special people should be responsible for the allocation.

\subsection{Selection and Training of Student Standardized Patient (SSP)}

4.4.1 Selection of SSP: in order to comply with development of OSCE in 2010 in the college, the college organized ten teachers from the Clinical Medical School in October 2009 to select SSP among the 350 students from the Chengbei Campus who were enrolled and who entered the college respectively in 2008 and 2009. They selected 100 students as the training targets for SSP from the 350 students.

\subsubsection{SSP training content}

4.4.2.1 According to training standard of SP in West China School of Medicine in Sichuan University, physicians and surgeons teachers from Clinical Medical School of LuZhou Medical College compiled the training scenario. The scenario was sent to students to enable them get familiar with the story 3-7d, during which period each major organized typical disease cases to let students vie and emulate and to experience emotion, language and body language of patients. Teachers further explained key points for simulation of a variety of disease groups.

\subsubsection{Inquiry about students and physical examination training among students}

After the strengthened training for three months, finally an evaluation was organized. According to students' mastery of the inquiry, standard of physical examination and life-like degree of simulation, 37 students were selected from the 100 students trained as the first batch of SSP for clinical skill evaluation.

\subsection{Arrangement of Examinees}

According to arrangement of the examination stations, the number of each batch of students and the examination time were confirmed, and then the examination date and time was arranged for all the examinees. In order to notify each examiner without any mistake, the college printed "OSCE time schedule" with the examination sequence, time, place and exam subjects and son on for the 622 students who entered the college in 2005.

\subsection{Meticulous Organization of Pre-examination}

In order to ensure successful development of OSCE, pre-examination of OSCE was necessary before the formal examination began after a meticulous preparation and planning. After the pre-examination was over, the college listened to opinions of students who participated in the evaluation about evaluation content and arrangement of examination station sites, organized teachers from all examination station sites to have discussion on problems emerging in the process of evaluation and asked for opinions of improvement.

OSCE pre-examination strengthened knowledge and understanding of the college in OSCE, encouraged students to actively find out their own disadvantages and guided a direction for further strengthening their clinical basic skills. At the same time, this pre-examination made a comprehensive test on standardized patients in the evaluation project, goods and materials guarantee, organization of personnel, alteration of station sites, performance statistics and so on, had great promoting effect on further strengthening the scientificalness, rationality and feasibility of the evaluation project and gained precious experience for formal implementation of OSCE.

\section{OSCE Flow in the College}

OSCE in the college is constituted by ten stations, including ten station sites, with the following content and score: 1) simulated diagnosis and treatment (50 minutes), 2) communication between doctors and patients (2 minutes), 3) surgeon skills (15 minutes), 4) medicine skills (10 minutes), 5) gynaecology and obstetrics skills (10 minutes), 6 ) cardiac auscultation (2 minutes), 7) lung auscultation (2 minutes), 8) screenage (3 minutes), 9) electrocardiogram (4 
minutes) and 10) biochemistry ( 2 minutes). The college classified the above 10 station sites into three major examination stations. The first station was simulated diagnosis and treatment station (including simulated diagnosis and treatment and communication between doctors and patients), the second station was basic skill operation station (including surgeon skills, medicine skills and gynaecology and obstetrics skills) and the third station was computer simulation examination station (including cardiac auscultation, lung auscultation, screenage, biochemistry and electrocardiogram). The examination was as below: the first station used SSP for disease history-taking (20 minutes), physical examination (18 minutes), case analysis (10 minutes), communication between doctors and patients (2 minutes) and questioning ( 2 minutes); the second station used simulated patients for basic skill operation of medicine, surgeon, gynaecology and obstetrics, and the third station used multi-media computer.

The examination hall was constituted by several examination stations, each station with independent rooms as the ward or consulting room. The examination hall was clearly marked, and at striking places in the examination hall were pasted schematic diagram of distribution of the examination hall, notice for examinees, examination flow and examination alteration diagram. Besides, each station site room was marked with numbers, in which way students could get familiar with the examination hall within a short period of time. Each station had a special guiding teaching to guide the examiners. The teachers asked each group of examiners to enter different stations (consulting rooms) according to the grouping of students set in advance. After having finished the previous station examination, examiners entered the next examination station in order according to direction of the guiding teachers.

\section{Analysis and Statistics of OSCE Performance}

This OSCE had altogether 621 students participating. There were 8 students with a total score lower than 60,74 students with a total score between 60-69, 304 students with a total score between 70-79, 225 students with a total score between 80-89 and 11 students with a total score above 90 . The examination performance was distributed in a positive direction.

\section{Improvement of OSCE}

OSCE is still at a trial and innovation phase in the college and we need to improve and enhance OSCE in the future from the following four aspects so as to make OSCE more rational, objective and justified.

1) To set up a mature SSP team, which requires to make efforts from the following two aspects.

A) To guarantee quality and set up perfect selection, training, management and application standard for standardized patients.

B) To strengthen communication and to hold fellowship activities.

2) To improve and enlarge the question bank. Design of the question bank should be cautious, with a view to evaluate students' judgment and treatment capacity in clinical issues with the theoretical knowledge they have learnt.

3) To improve the scoring sheet and station site setting mode and to enlarge generalization coefficient and reliability of the evaluation.

4) To strengthen the result feedback. After the examination is over, the evaluation result should be given back to students in time so as to help them improve their clinical skills.

In one word, to cultivate and improve medical students' comprehensive clinical capacity is the core of medical education. Only if the medical colleges continuously perfect the OSCE and enhance the teaching quality of clinical skills, can they achieve the target of cultivating high quality application-oriented talents.

\section{References}

Academic Department of Beijing LuSun Wyatt Co. Ltd. (2004). Application of Medical Simulation Education Abroad and Its Future Prospect in China. Medical Education, (5), 6

Burch VC, Nash RC, Zabow T., et a1. (2005). A structured assessment of newly qualified medical graduates. Med Educ, 39(7), 723

Deng, Shengli, Liang, Li, \& Hu, Weifeng. (2008). Several Experiences of Medical Students in Application of OSCE. Researches in Medical Education, 7(1), 61-62

Ding, Ke. (2001). Undergraduate Medical Education - Comparison of OSCEN. Standard Setting Method. Foreign Medical Sciences (Medical Education), 22(3), 1-4

Fumio Sano. (2000). OSCE in Japan. Progress in Japanese Medicine, 21(11), 526

Yang, Mianhua, He, Ping, Li, Liping et al. (2004). A first class clinics training center is an important base to train medical students in early medical training. China Higher Medical Education, (2), 50-51 